\title{
BMJ Open Dance PREEMIE, a Dance PaRticipation intervention for Extremely prEterm children with Motor Impairment at prEschool age: an Australian feasibility trial protocol
}

\author{
Kate L Cameron (D) , ${ }^{1,2}$ Jennifer L McGinley (D) , ${ }^{2}$ Kim Allison (D) , ${ }^{2}$ \\ Natalie A Fini (1) , 2,3 Jeanie L Y Cheong (1) , , 4 Alicia J Spittle (i) ${ }^{1,2,4}$
}

To cite: Cameron KL, McGinley JL, Allison K, et al. Dance PREEMIE, a Dance PaRticipation intervention for Extremely prEterm children with Motor Impairment at prEschool age: an Australian feasibility trial protocol. BMJ Open 2020;10:e034256. doi:10.1136/ bmjopen-2019-034256

- Prepublication history and additional material for this paper are available online. To view these files, please visit the journal online (http://dx.doi org/10.1136/bmjopen-2019034256).

Received 16 September 2019 Revised 28 November 2019 Accepted 07 January 2020

Check for updates

(C) Author(s) (or their employer(s)) 2020. Re-use permitted under CC BY-NC. No commercial re-use. See rights and permissions. Published by BMJ.

For numbered affiliations see end of article.

Correspondence to

Kate L Cameron;

kate.cameron@mcri.edu.au

\section{ABSTRACT}

Introduction Children born extremely preterm (EP: $<28$ weeks gestation) and/or extremely low birth weight (ELBW: $<1000 \mathrm{~g}$ ) are at increased risk of motor impairment compared with children born at term. Children with motor impairment have lower rates of physical activity (PA) participation compared with their typically developing peers. PA participation is an important outcome for children with motor impairment, however, there is limited evidence available to support interventions that improve PA participation in this population. The aim of this study is to assess the feasibility, including the recruitment and retention, acceptability and fidelity, of a preschool dance participation intervention for children born EP/EBLW with motor impairment called Dance PaRticipation intervention for Extremely prEterm children with Motor Impairment at prEschool age.

Methods and analysis This feasibility case series trial will recruit EP/ELBW children with motor impairment $(n=10)$ from the Victorian Infant Collaborative Study 2016/2017 cohort, a prospective longitudinal cohort study. Up to 10 community-based dance teachers will be recruited and provided with physiotherapy-led training and support to facilitate the participation of EP/ELBW children in community dance classes. A mixed-methods approach (quantitative and qualitative) will be used to analyse the primary aim, to determine the feasibility of the intervention from the perspectives of families and dance teachers. Ethics and dissemination This study is approved by the Human Research Ethics Committees of The Royal Children's Hospital and The Royal Women's Hospital, Melbourne. Study outcomes will be disseminated through conference presentations, peer-reviewed publications and social media.

Trial registration number ACTRN12619001266156

\section{INTRODUCTION}

Children born extremely preterm (EP: $<28$ weeks gestation) and/or extremely low birth weight (ELBW: $<1000 \mathrm{~g}$ ) are at increased risk of motor impairment compared with children born at term; approximately 15\%

\section{Strengths and limitations of this study}

- This is a unique intervention aiming to promote physical activity participation for children born extremely preterm/extremely low birthweight.

- Recruiting and upskilling community providers of physical education (dance teachers) to promote physical activity participation in a preschool population with motor impairment is a novel intervention approach.

- A mixed-methods focus on the acceptability of the intervention will provide valuable insight into the barriers and facilitators to implementing participation interventions in a community setting.

- Participant numbers (10 children) were chosen for pragmatic reasons and to test feasibility to inform future trials.

- The study will not be sufficiently powered to detect changes in motor performance and/or participation.

of children born EP will be diagnosed with cerebral palsy (CP) and $50 \%$ will have non-CP motor impairment, such as developmental coordination disorder (DCD). ${ }^{1}{ }^{2}$ With the implementation of new interventions in the neonatal intensive care unit, survival rates of children born EP/ELBW have increased over the past two decades, ${ }^{3}$ however, there is growing evidence that rates of motor impairment in the preterm cohort are not declining concurrently as expected. ${ }^{5}$ Of concern, there appears to be a trend of increasing non-CP motor impairment in this population. ${ }^{5}$ Children with motor impairment often experience poor self-efficacy and low levels of physical activity (PA) participation as they reach school age and adolescence ${ }^{67}$ Current evidence suggests that motor impairment in children born EP persists throughout 
childhood and adolescence, with relevance for lifelong PA participation and associated health outcomes. ${ }^{8}$

At preschool age, children are learning and refining a range of motor skills, which provide the critical foundation for the development of more complex motor skills, ${ }^{9}$ and facilitate participation in lifelong PA. ${ }^{10}$ Delay or impairment in acquiring these motor skills has been associated with lower levels of PA participation throughout adolescence and into adulthood. ${ }^{11}$ Preschool age is an ideal period to develop motor skills through participation in structured, age appropriate, PA. ${ }^{9}$ Dance involves structured movement in all planes and develops balance, ${ }^{12}$ postural control, proprioception and selective motor control. It is repetitive and progressive, and thus facilitates learning and mastery of motor skills. ${ }^{13}$ Furthermore, dance has been shown to have social and cognitive benefits for children, including children with social or intellectual difficulties, such as autism spectrum disorder. ${ }^{14}$ With approximately $50 \%$ of EP/ELBW children presenting with motor impairment at school age, ${ }^{1}$ it is important to consider options for promoting motor skill learning and PA participation in this population.

Participation is an important outcome for children with motor impairment. ${ }^{15}$ Participation can be defined as 'involvement in a life situation' and describes a child's attendance and involvement within a social context; participating in a PA class for example. ${ }^{16}$ By school age, children with motor impairment have lower levels of participation than their typically developing peers. ${ }^{17}$ This is of concern, as not only is participation a meaningful outcome, but it provides opportunity for children to develop and perform motor skills in a real-world context, rather than simply the capacity to complete a skill in a clinical environment. ${ }^{18}$ Interventions for children with motor impairment commonly focus on motor skill acquisition, ${ }^{19}$ however, there is increasing recognition that improvement in physical capacity does not invariably lead to improved participation. ${ }^{152021}$ It is, therefore, imperative to develop interventions that directly aim to promote participation. Although PA participation is considered an important outcome for children with motor impairment, ${ }^{15}$ there is limited evidence available to support participation interventions in this population. ${ }^{19}$

The goal of Dance PREEMIE (Dance PaRticipation intervention for Extremely prEterm children with Motor Impairment at prEschool age) is to promote PA participation for preschool age children born EP/ELBW with motor impairment through facilitating participation in community dance classes taught by teachers provided with physiotherapy-led training. Implementing an intervention aiming to improve participation is likely to be more effective if it can be provided in a way that takes barriers and facilitators into account and prioritises the needs of children and their family. For younger children in particular, participation is significantly influenced by a child's environment and family context. ${ }^{16}$ Therefore, the proposed study will focus on the feasibility, including acceptability and fidelity, of the intervention to the participating children and families. In addition, through gaining the perspectives of community dance teachers, this study will provide valuable knowledge on the process of collaborating and educating beyond the clinical setting. This study, therefore, aims to assess the feasibility of a preschool dance participation intervention for children born EP/ELBW with motor impairment.

\section{Objectives}

The aim of this study is to determine the feasibility of a dance participation intervention for preschool age children (3 years corrected age) born EP/ELBW with motor impairment including recruitment feasibility, acceptability and implementation fidelity. The specific aims are to determine:

1. The effectiveness of study recruitment strategies to enrol sufficient participants, both preschool age children born EP/ELBW and dance teachers.

2. The acceptability of the intervention, including baseline and follow-up assessment and number of classes attended, to participating preschool age children born $\mathrm{EP} / \mathrm{ELBW}$ and their families.

3. The acceptability of the intervention procedure, including training and follow-up, to participating dance teachers.

4. Barriers and facilitators to participation in dance for preschool age children born EP/ELBW and their families.

5. To obtain insight into the feasibility of measuring the implementation fidelity of the dance intervention.

\section{Administrative information}

Protocol V.7: dated 11 September 2019

Study sponsor: Murdoch Children's Research Institute, 50 Flemington Rd, Parkville, Victoria, Australia, 3052.

\section{METHODS AND ANALYSIS \\ Design}

This study is a feasibility trial of a mixed-methods (qualitative and quantitative) case series.

\section{Participants}

1. Children born EP/ELBW with motor impairment.

2. Community dance teachers.

\section{Eligibility criteria: children born EP/ELBW}

Children will be recruited from the Victorian Infant Collaborative Study (VICS) 2016/2017 cohort, a longitudinal geographical cohort study of children born EP and/or ELBW and term controls in Victoria, Australia. ${ }^{22}$ As part of the larger VICS study, children are assessed at 2 years corrected age using the Bayley Scales of Toddler and Infant Development, Third Edition (Bayley III), which provides norm referenced scores and percentiles across five developmental domains, including for motor development. ${ }^{23}$ Based on results of previous cohorts, approximately $50 \%$ of EP/ELBW children will be considered at risk of motor impairment. ${ }^{24}$ Children in the 
EP/ELBW group of VICS who were born at the Royal Women's Hospital (RWH), and who have motor impairment at 2 years (corrected) age according to the Bayley III, will be eligible for inclusion in the study. For families with multiple children enrolled in VICS (eg, families with twins), all children will be considered eligible for Dance PREEMIE if one or more child/children meets inclusion criteria.

\section{Inclusion criteria}

1. Children with motor impairment, defined as scoring $>1$ SD below the mean (established by the VICS 2005 term control cohort; mean 118.4 SD 16.7) ${ }^{24}$ on the mean motor score of the Bayley III Scale of Infant and Toddler Development at 2-year follow-up. A mean value established from a contemporary cohort of healthy, term born Australian children will decrease the likelihood the Bayley III will under predict motor impairment.

\section{Exclusion criteria}

1. Children who are unable to consistently follow simple commands (due to global developmental delay, receptive language disorders).

2. Children who are non-ambulant.

3. Children who have any medical condition that precludes participation in PA.

\section{Recruitment: children born EP/ELBW}

Children enrolled in the VICS 2016/2017 cohort who were born at RWH are invited to attend a 2-year neurodevelopmental assessment at either RWH or Murdoch Children's Research Institute (MCRI) in Melbourne, Australia. At this time, families of children born EP/ ELBW will have the Dance PREEMIE study briefly explained to them by a research nurse who is known to the families from previous assessments. The research nurses will be educated on the Dance PREEMIE study by the investigator-KLC and will be given an information sheet to ensure information provided to families is consistent. Families will be provided with a paper or electronic copy of the Dance PREEMIE information sheet and a permission to contact form when they attend the 2-year assessment. Permission to contact forms can be completed on the day of assessment or returned via email. Families will be provided with the contact details of investigator-KLC if they wish to ask further questions. All families who fill out a permission to contact form will be contacted by investigator-KLC to inform the family whether or not their child fits the eligibility criteria. Investigator-KLC will explain to eligible families that they will be next contacted when their child approaches 3 years corrected age to obtain informed consent. The recruitment process for Dance PREEMIE is designed to fit within standard follow-up for children born EP/ELBW in Victoria, Australia; all children are invited to attend a neurodevelopmental assessment at 2 years corrected age. Dance PREEMIE uses motor skills assessment scores gained at this assessment in order to (1) decrease burden on families by limiting the number of assessments they need to attend and (2) decrease the costs involved with implementing the study.

\section{Informed consent: children born EP/ELBW}

Eligible families will be contacted by telephone by investigator-KLC in either term 32019 (if the child will be 3 years corrected age by 7 October 2019) or in term 42019 (if the child will be 3 years corrected age by 29 January 2020). A screening process will ensure children fulfil eligibility criteria. Motor skills will not be reassessed as part of the recruitment process. If a child has a medical condition that may preclude participation in the study, the child's general practitioner (GP) or paediatrician will be contacted (with parental consent) to gain approval to participate. Families will be provided with written information in the form of a plain language participant information and consent form (online supplementary material S1: parent information and consent form), as well as verbal information from a member of the research team. If parents agree to be involved in the study, they will be asked to sign this consent form. Participants may withdraw from the study at any time. This intervention is designed to promote PA participation and does not replace physiotherapy, therefore, this trial places no restrictions on concurrent therapy (eg, physiotherapy) children access throughout the intervention.

\section{Eligibility criteria: dance teachers}

Preschool dance teachers in metropolitan Melbourne will be eligible for participation.

\section{Inclusion criteria}

Dance teachers must:

1. Be teaching a preschool dance class.

2. Be able to attend a training session at MCRI.

3. Have a valid working with children (WWC) check.

4. Have a valid first aid certificate (or have a staff member in the studio who is the designated first aider).

\section{Exclusion criteria}

Dance classes must not:

1. Be outside $100 \mathrm{~km}$ radius of MCRI.

2. Be conducted in a language other than English.

\section{Recruitment: dance teachers}

Dance schools and teachers across metropolitan Melbourne will be approached via email or telephone (contact details of dance schools are publicly available). Dance teachers will be provided with written and verbal information about the study and encouraged to ask questions. Eligibility will be confirmed via telephone screening. Due to Melbourne's large geographical area, we estimate $8-10$ dance teachers will be recruited to enable dance classes to be within an acceptable distance for the participating families. 


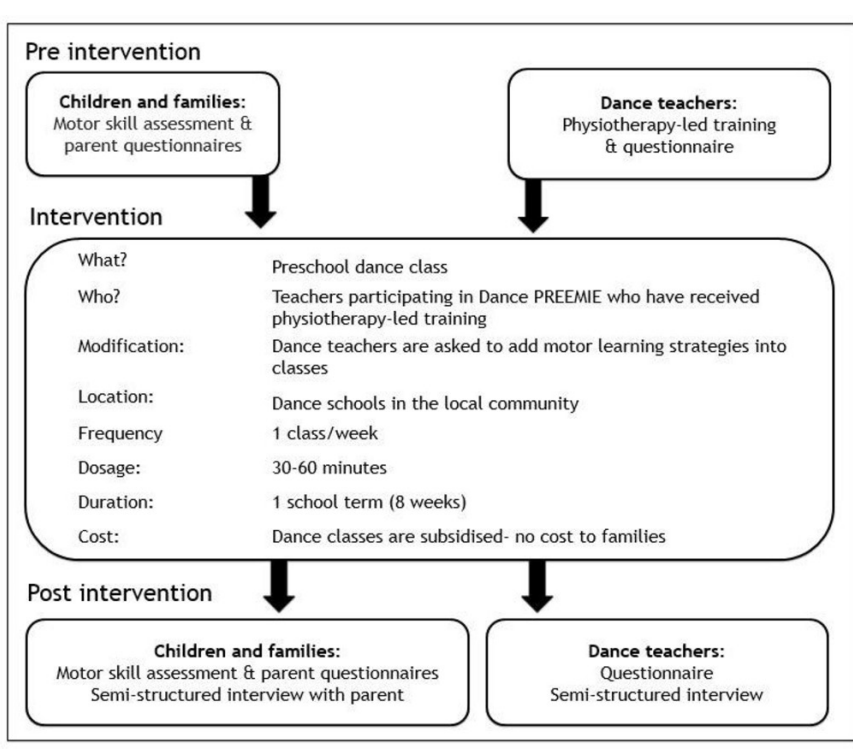

Figure 1 Study procedure. Dance PREEMIE, Dance

PaRticipation intervention for Extremely prEterm children with Motor Impairment at prEschool age.

\section{Informed consent: dance teachers}

Eligible and interested dance teachers will be provided with further written information about the study in the form of a participant information and consent form (online supplementary material S2: teacher information and consent form), and will be given the opportunity to ask questions during telephone screening with investigator-KLC. If dance teachers choose to be involved, they will be asked to sign this consent form. Dance teachers may withdraw from the study at any time. In addition, dance teachers will be asked to provide proof of WWC and first aid certificate. Dance school owners will be asked to sign a letter of agreement. This letter of agreement outlines the responsibilities of the dance school and will allow the study to subsidise dance school fees.

\section{INTERVENTION AND PROCEDURE}

The study procedure is outlined in figure 1 . The study procedure will be repeated twice; during Australian school term 4, 2019 (October to December) and term 1, 2020 (February to April).

\section{Dance teacher training}

All participating dance teachers will receive physiotherapy-led face-to-face training on teaching children with motor impairments and motor learning principles. The purpose of the training session is to provide participating dance teachers with a range of evidence-based strategies to use throughout the intervention period. The training session will begin with a brief overview of preterm birth, as well as common diagnoses involving motor impairment in this population, including $\mathrm{CP}$ and DCD. The training will then focus on how children learn movement through discussing the stages of motor learning (Fitts and Posner model),${ }^{25}$ the interaction between child, task and environment and the importance of having just the right level of challenge. ${ }^{26}$ Strategies for adjusting the task or environment to suit the child's ability and stage of learning will then be introduced using the M.A.T.C.H. framework, developed by CanChild. ${ }^{27}$ Teachers will be encouraged to use strategies that reflect motor learning principles, including specificity, repetition and salience. Use of appropriate modelling and feedback types and frequency suitable for children will also be discussed. ${ }^{28}$ It is anticipated that dance teachers will already use many of these strategies in their classrooms. Our training session is unique in that it aims to discuss these teaching strategies in the context of children born EP/ELBW who may have challenges with learning movements or maintaining attention compared with typically developing children. Participating teachers will be encouraged to engage with the training content through activities interspersed throughout the session. These activities will include group discussion, practice of teaching techniques in pairs and applying the M.A.T.C.H. framework using video recordings of children in dance classes as a starting point. Physiotherapy-led training sessions will be approximately 3-4 hours in duration and will take place at MCRI prior to the commencement of Australian school term 4, 2019 and term 1, 2020. Participating dance teachers are only required to attend one session. A single training session was chosen for pragmatic reasons and to limit burden on participating dance teachers. The training will be delivered in a group setting and delivered by investigators-KLC and NAF. In addition, dance teachers will be provided with supporting material in the form of a printed educational package (online supplementary material S3: dance teacher training manual). Investigator-KLC will be contactable throughout the study in order to assist with any queries or provide additional support for the participating dance teachers.

\section{Dance classes}

Once informed consent is obtained, children will be matched with a dance class within an acceptable distance of the family's home. Children will be enrolled to attend the dance class once per week for 8 weeks. This time frame was chosen to fit within an Australian school term. If the term is longer than 8 weeks, the child can attend the full term, however, all post-intervention assessments will take place after 8 weeks of class. Dance classes are likely to be variable in duration and intensity but must be between 30 and $60 \mathrm{~min}$. Dance class fees will be fully subsidised to facilitate involvement in the study and minimise financial barriers to participation. Children will be asked to rate their enjoyment after each class using a five-point ordinal smiley face scale. ${ }^{29}$ Parents will be asked to administer the scale by asking their child what they thought about the class and recording their child's response (from 1, very sad face/awful to 5 , very happy face/fantastic) on a paper form and bring the completed form to their final assessment. 


\section{Implementation fidelity}

Implementation fidelity refers to the extent to which individuals (dance teachers) implement the intervention protocol (adherence) and the skilfulness of delivery (competence). ${ }^{30}$ Implementation fidelity will be monitored through observation performance evaluation using a predetermined checklist (online supplementary material S4: implementation fidelity checklist) based on the education session content. This method was successfully used by the SKIPing study, in which motor skill experts provided education to physical education teachers. ${ }^{31}$ There is no reliability data for our checklist as it is new tool, however, we have chosen to measure fidelity through direct observation in order to minimise bias; observational tools have been found to be more accurate than self-reported measures. ${ }^{30}$ No existing implementation fidelity tools were appropriate for our study. Participating dance teachers will be informed that a member of the research team will observe their class. The same member of the research team, investigator-KLC, will complete all observation in order to minimise inter-rater bias. Each dance class will be observed twice throughout the intervention to ensure key components discussed in the education session are implemented during classes. Observation time points will be chosen pragmatically based on the availability of the investigator and the time and location of the dance class.

\section{Assessment}

Dance teachers

At the physiotherapy-led training session, teachers will be asked to complete a questionnaire before and immediately after the education is delivered (online supplementary material S5: teacher questionnaire). Teachers will be asked to rate their agreement using a 10-point Likert scale (from 'strongly disagree' to 'strongly agree') to a series of statements about their confidence and knowledge on teaching children with motor impairment. After each dance class, the teacher will complete an attendance form, indicating if the child attended the class as well as rating the child's involvement using a 5-point Likert scale (from 1 'not at all involved' to 5 'very involved'). Teachers will be asked to return this form at the end of the intervention period. Post-intervention, dance teachers will rate their own self-efficacy a third time (online supplementary material S5: teacher questionnaire), as well as participate in a semistructured telephone interview exploring their experiences with the dance training and intervention.

\section{Children born EP/ELBW and families}

Children will attend the Royal Children's Hospital for a baseline (pre-intervention) and post-intervention assessment (after 8 weeks of dance classes). Assessment measures are outlined in table 1 . The motor skills assessment and goal setting will be completed and scored by a qualified and experienced physiotherapist. Other than the Canadian Occupational Performance Measure (COPM) goals, test results from the baseline assessment will not be accessible to the assessor at the post-intervention assessment. In

Table 1 Child assessment measures

\begin{tabular}{|c|c|c|}
\hline Assessment & Description & Assessment schedule \\
\hline $\begin{array}{l}\text { The Movement Assessment } \\
\text { Battery for Children, second } \\
\text { edition (MABC-2). }{ }^{37}\end{array}$ & $\begin{array}{l}\text { The MABC- } 2 \text { is considered the gold standard at } \\
\text { assessing for motor impairment for children } 3-16 \text { years } \\
\text { of age. }{ }^{38} \text { The assessment is divided into subscales; } \\
\text { manual dexterity, aiming and catching and balance. }\end{array}$ & $\begin{array}{l}\text { Baseline } \\
\text { Post-intervention }\end{array}$ \\
\hline $\begin{array}{l}\text { Preschool-age Physical Activity } \\
\text { Questionnaire. }\end{array}$ & $\begin{array}{l}\text { This validated questionnaire was developed in } \\
\text { an Australian context and is designed to capture } \\
\text { information on sedentary behaviour and physical activity } \\
\text { time, including structured physical activity. }\end{array}$ & $\begin{array}{l}\text { Baseline } \\
\text { Post-intervention } \\
3 \text { months post-intervention }\end{array}$ \\
\hline $\begin{array}{l}\text { Canadian Occupational } \\
\text { Performance Measure (COPM). }{ }^{40}\end{array}$ & $\begin{array}{l}\text { The physiotherapist assessor will work collaboratively } \\
\text { with the parent to develop and prioritise gross motor } \\
\text { and PA participation goals during the pre-intervention } \\
\text { assessment. Parent's will be asked to rate their } \\
\text { child's performance and satisfaction with their child's } \\
\text { performance for each goal. }\end{array}$ & $\begin{array}{l}\text { Baseline } \\
\text { Post-intervention }\end{array}$ \\
\hline $\begin{array}{l}\text { Strengths and Difficulties } \\
\text { Questionnaire. }{ }^{41}\end{array}$ & $\begin{array}{l}\text { This questionnaire provides information about emotional } \\
\text { symptoms, conduct problems, hyperactivity/inattention, } \\
\text { peer relationship problems and prosocial behaviour. }\end{array}$ & Baseline \\
\hline Smiley Face Scale & $\begin{array}{l}\text { 5-point Likert scale of smiley faces used to assess } \\
\text { enjoyment in children. Previously used in intervention } \\
\text { studies for children. }{ }^{29}\end{array}$ & $\begin{array}{l}\text { Each dance lesson attended } \\
\text { (completed by child and parent) }\end{array}$ \\
\hline $\begin{array}{l}\text { Attendance and Involvement } \\
\text { Scale }\end{array}$ & $\begin{array}{l}\text { Weekly record of child attendance in class as well as a } \\
\text { rating of the child's involvement on a 5-point Likert scale } \\
\text { from 'not at all involved' to 'very involved'. }\end{array}$ & $\begin{array}{l}\text { Each dance lesson attended } \\
\text { (completed by teacher) }\end{array}$ \\
\hline
\end{tabular}


addition, participant characteristics, including sex, perinatal characteristics, parental socioeconomic status and Bayley III results, will be obtained from the VICS study. At the time of the post-intervention assessment, or via telephone after the assessment, parent(s) will participate in an interview to discuss the acceptability and accessibility of the dance trial. Data will be collected for each participating child with the exception of the semistructured interview, where only one interview per family will be conducted to minimise burden to parents with multiple participating children.

\section{Feedback to families}

The results of the initial Movement Assessment Battery for Children, second edition (MABC-2) assessment will be provided to families in the form of a written report. This will be posted to parents, along with a thank you card. Families will be encouraged to share the results of the report with their GP or paediatrician.

\section{Interviews}

Both parents and dance teachers will be invited to participate in interviews after the intervention in order to gain a rich understanding of the acceptability of the study. Interviews will be semistructured and theoretically based, to enable the exploration of participants' experiences and viewpoints, with the aim of collecting meaningful data to inform refinements to the intervention. The semistructured interview guide will be developed in line with the Theoretical Framework of Acceptability (TFA). ${ }^{32}$ The TFA starts from the position that the effectiveness of any intervention (or the efficacy of an intervention in a realworld situation) is dependent on how acceptable the intervention is, and further, that the intervention needs to be considered acceptable by those delivering as well as receiving the intervention. ${ }^{32}$ The TFA proposes seven constructs of acceptability; affective attitude, burden, ethicality, intervention coherence, opportunity costs, perceived effectiveness and self-efficacy, ${ }^{32}$ which will all be explored in the interview process. For parents, the semistructured interview will specifically seek to explore: (1) the acceptability of the intervention, including the baseline and post-intervention assessments, (2) the barriers and facilitators to attendance of dance classes and involvement in the intervention and (3) the perceived effect of the intervention. Thorough exploration of intervention feasibility from a parent perspective will facilitate the development of PA participation programmes that are acceptable to families with a child born EP/ELBW. For dance teachers, the interviews will seek to explore: (1) the acceptability of the training and intervention, (2) the effect of the training session and the inclusion of an EP/ELBW child on participants' teaching practice, including an understanding of the dance teacher experience and (3) the barriers and facilitators to intervention implementation. Interviews will be $30-40 \mathrm{~min}$ in duration, audio recorded and transcribed by an external provider. Semi structured interview scripts are provided as supplementary material (online supplementary material S6 semistructured interview scripts). Analysis will be performed concurrently to facilitate a constant comparative approach in the thematic analysis, and therefore, incrementally identify, synthesise and contrast ideas identified from the data. ${ }^{33}$ To enhance credibility, thematic analysis will be performed independently by two investigators trained in qualitative research methods.

\section{Feasibility outcomes}

For the purposes of this study, we consider feasibility to include; recruitment and retention capability, implementation fidelity and study acceptability. Study acceptability will be assessed through the use of semistructured interviews (Interviews) and a smiley face scale (table 1). Implementation fidelity will be assessed through observation. Feasibility will also be assessed through quantitative measures including: (1) recruitment rates of eligible children, (2) recruitment rates of dance schools, (3) dance class attendance rates of children (4) retention and follow-up rates of all participants.

\section{Sample size estimation}

This feasibility study has chosen to use a sample size of $10 \mathrm{EP} / \mathrm{ELBW}$ children and up to 10 dance class teachers on pragmatic grounds. The data obtained from the small samples in the feasibility study will help us to determine sample sizes for a larger randomised trial of Dance PREEMIE, if a large trial proves to be feasible in the future.

\section{Data management}

All assessment measures will be entered into an electronic REDCap (Research Electronic Data Capture) database by a member of the research team, except for the Preschool-age Physical Activity Questionnaire (Pre-PAQ) (parents) and teacher questionnaire (teachers) which will be completed as online surveys through REDCap.

\section{Adverse events and risks}

We do not predict any risks for children or dance teachers participating in this study. Inclusion in this study does not prevent children and their families from accessing any other forms of physiotherapy or other intervention.

\section{Methods of analysis}

Descriptive data will be used to assess participant characteristics, recruitment rates of children and dance teachers, as well as rates of class attendance and follow-up. For continuous data (eg, MABC-2), results will be expressed as mean, SD and CI. For measures with two time points (eg, MABC-2 and COPM), mean of the score differences will be reported along with the SD of differences and the $95 \%$ CI. Linear regression, fitted with generalising estimating equations to account for twins, will assess changes in PA (Pre-PAQ) between pre-intervention and post-intervention, and post-intervention and 3 months follow-up. The study is not powered to detect differences 
in these measures but will enable effect sizes for future research to be determined.

Intervention fidelity will be reported using descriptive statistics. The cumulative score for each teacher (from two observations) will be calculated, with each key component scored as adhered to (1) or not adhered to $(0)$, and expressed as a percentage. Values above $50 \%$ will be considered acceptable implementation fidelity. ${ }^{31}$

For the qualitative data, thematic analysis will be performed independently by two members of the research team using inductive analysis. To enhance credibility and trustworthiness of the work, all themes will be reviewed by all members of the research team, four of whom have clinical and research experience in paediatrics and two who have diverse qualitative research experience.

\section{Patient and public involvement}

Patient feedback has been built into the study design as one of its primary outcomes; acceptability. The semistructured interviews are designed to gain rich data on the acceptability of the study from the perspective of those delivering the intervention (dance teachers) as well as the families receiving the intervention. The views and experiences of the dance teachers and families involved in this study will inform the study designs of future interventions aiming to promote participation, including a larger randomised controlled trial (RCT) of this study if feasible. There was no direct involvement of dance teachers, EP/ EBLW individuals or their families in the development of the research question or feasibility study design.

\section{Ethics and dissemination}

Study results will be compiled and written up into submissions to peer-reviewed medical and developmental journals, and will form part of investigator-KLC's PhD thesis. Study results may also be submitted to relevant conferences. Families will be sent a written report detailing the results of their child's motor assessment. In addition, all participating families and dance teachers will be sent a report at the completion of the study, which will outline the results and thank participants for their involvement. No further amendments to this protocol are planned.

\section{DISCUSSION}

This study is a unique intervention in two distinct ways. First, it aims to promote PA participation in children born EP/ELBW with motor impairment. There are very few studies that focus on participation as an outcome, ${ }^{19}$ and none that promote participation for this population. Participation is recognised as a meaningful outcome for children with motor impairment. ${ }^{15}$ Clinicians are encouraged to promote participation, ${ }^{34}$ however, there is little evidence available to support them. Second, this intervention employs a novel study design by upskilling community providers of PA, rather than choosing to have therapists or other healthcare providers deliver the intervention. This decision was made with the intent of providing an intervention that used participation as a means, as well as an end. ${ }^{15}$ Through recruiting dance teachers with existing community dance classes, this study facilitates participation and inclusion in real world situations for the children enrolled in this study. By enabling children to participate with peers, we are hoping to create an inclusive environment to facilitate ongoing PA participation.

Feasibility studies, by definition, ask whether or not an intervention can be done. In contrast to an RCT, or other study evaluating the outcomes of the intervention, a feasibility study focuses on the process of implementing the intervention, and the acceptability of this process to those involved.$^{35}$ As this study uses novel intervention design, a feasibility study is warranted to ensure that the study process is both possible and acceptable before moving on to an RCT. There are several key challenges within this study design that make a feasibility study a prudent choice. First, the recruitment of two sets of participants; dance teachers and children born EP/ELBW. Recruiting the teachers that will deliver the intervention as well as the children that will receive the intervention elevates the importance of evaluating the recruitment strategy, as recruitment capability of the dance teacher group will affect the involvement of the recruited EP/ELBW group. Furthermore, the task of matching participating children with dance classes at a time and place that suits the family provides a second recruitment challenge that makes this study best suited to a feasibility design. While assessment of study process provides information on what is possible to achieve in terms of recruitment and delivery, another important aspect of feasibility studies is the acceptability of the study to both those receiving and delivering the intervention. ${ }^{36}$ The use of semistructured interviews to qualitatively assess the acceptability of the intervention design will provide valuable information on the experiences of both participating dance teachers and families of children born EP/ELBW. Through using a feasibility design, this study will assess whether the Dance PREEMIE intervention is achievable and acceptable. Furthermore, this study will gain valuable insight into the process of implementing participation-centred interventions, which may assist in design and development of similar interventions in the future.

\section{Author affiliations}

${ }^{1}$ Victorian Infant Brain Studies, Murdoch Childrens Research Institute, Parkville, Victoria, Australia

${ }^{2}$ Physiotherapy, University of Melbourne, Parkville, Victoria, Australia

${ }^{3}$ Physiotherapy, Alfred Health, Melbourne, Victoria, Australia

${ }^{4}$ Newborn Research, Royal Women's Hospital, Parkville, Victoria, Australia

${ }^{5}$ Obstetrics and Gynaecology, University of Melbourne, Parkville, Victoria, Australia

Twitter Kate L Cameron @kate_cameron and Natalie A Fini @NatFiniPhysio

Contributors Author contributions: KLC conceived and designed the study, drafted and critically revised the manuscript and gave the final approval of the version to be published. JLM, NAF, KA, JLYC and AJS conceived and designed the study, critically reviewed the manuscript and gave the final approval of the version to be published.

Funding This work is supported by grants from the Physiotherapy Research Foundation, Australia (Project grant S18-021), the National Health and Medical Research Council of Australia (Centre of Research Excellence \#1060733 
and \#1153176; Career Development Fellowship \#1141354 to JLYC; Career Development Fellowship \#1108714 to AJS), and the Victorian Government's Operational Infrastructure Support Program. KLC's PhD candidature is supported by The Australian Government Research Training Program Scholarship and the Centre of Research Excellence in Newborn Medicine.

Disclaimer The funding sources had no role in the study design.

Competing interests None declared.

Patient consent for publication Not required.

Ethics approval This study has ethical approval from the Royal Children's Hospital and the Royal Women's Hospital.

Provenance and peer review Not commissioned; externally peer reviewed.

Open access This is an open access article distributed in accordance with the Creative Commons Attribution Non Commercial (CC BY-NC 4.0) license, which permits others to distribute, remix, adapt, build upon this work non-commercially, and license their derivative works on different terms, provided the original work is properly cited, appropriate credit is given, any changes made indicated, and the use is non-commercial. See: http://creativecommons.org/licenses/by-nc/4.0/.

\section{ORCID iDs}

Kate L Cameron http://orcid.org/0000-0001-5447-594X

Jennifer L McGinley http://orcid.org/0000-0003-3775-9267

Kim Allison https://orcid.org/0000-0003-1344-1465

Natalie A Fini http://orcid.org/0000-0001-5474-6404

Jeanie L Y Cheong https://orcid.org/0000-0001-5901-0455

Alicia J Spittle http://orcid.org/0000-0002-6535-661X

\section{REFERENCES}

1 Williams J, Lee KJ, Anderson PJ. Prevalence of motor-skill impairment in preterm children who do not develop cerebral palsy: a systematic review. Dev Med Child Neurol 2010;52:232-7.

2 Himpens E, Van den Broeck C, Oostra A, et al. Prevalence, type, distribution, and severity of cerebral palsy in relation to gestational age: a meta-analytic review. Dev Med Child Neurol 2008;50:334-40.

3 Seaton SE, King S, Manktelow BN, et al. Babies born at the threshold of viability: changes in survival and workload over 20 years. Arch Dis Child Fetal Neonatal Ed 2013:98:F15-20.

4 Cheong JLY, Anderson PJ, Burnett AC, et al. Changing neurodevelopment at 8 years in children born extremely preterm since the 1990s. Pediatrics 2017;139:6-8.

5 Spittle AJ, Cameron K, Doyle LW, et al. Motor impairment trends in extremely preterm children. Pediatrics 2018:1991-2005.

6 Cairney J, Hay JA, Faught BE, et al. Developmental coordination disorder, generalized self-efficacy toward physical activity, and participation in organized and free play activities. $J$ Pediatr 2005;147:515-20.

7 Dahan-Oliel N, Mazer B, Majnemer A. Preterm birth and leisure participation: a synthesis of the literature. Res Dev Disabil 2012;33:1211-20.

8 de Kieviet JF, Piek JP, Aarnoudse-Moens CS, et al. Motor development in very preterm and very low-birth-weight children from birth to adolescence. JAMA 2009;302:2235-42.

9 Gallahue DL, Goodway J, Ozmun JC. Understanding motor development : infants, children, adolescents, adults. 7th ed. New York: McGraw-Hill, 2012.

10 Lubans DR, Morgan PJ, Cliff DP, et al. Fundamental movement skills in children and adolescents review of associated health benefits. Sports Med 2010;40:1019-35.

11 Barnett LM, van Beurden E, Morgan PJ, et al. Childhood motor skill proficiency as a predictor of adolescent physical activity. $J$ Adolesc Health 2009;44:252-9.

12 Fong Yan A, Cobley S, Chan C, et al. The effectiveness of dance interventions on physical health outcomes compared to other forms of physical activity: a systematic review and meta-analysis. Sports med 2017.

13 López-Ortiz C, Gladden K, Deon L, et al. Dance program for physical rehabilitation and participation in children with cerebral palsy. Arts Health 2012;4:39-54.

14 Nelson C, Paul K, Johnston SS, et al. Use of a creative dance intervention package to increase social engagement and play complexity of young children with autism spectrum disorder. Educ Train Autism De 2017:52:170-85.

15 Imms C, Granlund M, Wilson PH, et al. Participation, both a means and an end: a conceptual analysis of processes and outcomes in childhood disability. Dev Med Child Neurol 2017;59:16-25.
16 World Health Organisation. ICF-CY, International Classification of Functioning, Disability, and Health: Children \& Youth version. Geneva: World Health Organisation, 2007.

17 Jarus T, Lourie-Gelberg Y, Engel-Yeger B, et al. Participation patterns of school-aged children with and without DCD. Res Dev Disabil 2011;32:1323-31.

18 Palisano RJ, Chiarello LA, King GA, et al. Participation-based therapy for children with physical disabilities. Disabil Rehabil 2012;34:1041-52.

19 Adair B, Ullenhag A, Keen D, et al. The effect of interventions aimed at improving participation outcomes for children with disabilities: a systematic review. Dev Med Child Neurol 2015;57:1093-104.

20 Wright FV, Rosenbaum PL, Goldsmith $\mathrm{CH}$, et al. How do changes in body functions and structures, activity, and participation relate in children with cerebral palsy? Dev Med Child Neurol 2008;50:283-9.

21 Blank R, Smits-Engelsman B, Polatajko H, et al. European Academy for Childhood Disability (EACD): Recommendations on the definition, diagnosis and intervention of developmental coordination disorder (long version)*. Dev Med Child Neurol 2012;54:54-93.

22 Spittle AJ, McGinley JL, Thompson D, et al. Motor trajectories from birth to 5 years of children born at less than 30 weeks' gestation: early predictors and functional implications. Protocol for a prospective cohort study. J Physiother 2016;62:222-3.

23 Bayley N. Bayley scales of infant and toddler development. New York: Psychological Corporation, 2005.

24 Anderson PJ, De Luca CR, Hutchinson E, et al. Victorian infant collaborative $\mathrm{G}$. underestimation of developmental delay by the new Bayley-III scale. Arch Pediat Adol Med 2010;164:352-6.

25 Fitts PM, Posner MI. Human performance. Brooks/Cole Pub. Co, 1967.

26 Guadagnoli MA, Lee TD. Challenge point: a framework for conceptualizing the effects of various practice conditions in motor learning. J Mot Behav 2004;36:212-24.

27 Missiuna C, Rivard L, Pollock N. They're Bright but Can't Write: Developmental Coordination Disorder in School Aged Children. Teaching Except Children Plus 2004;1.

28 Gordon A, Magill R. Motor learning: Application of Principles to Pediatric Rehabilitation. In: Palisano R, Orlin M, Schrieber J, eds. Campbell's physical therapy for children. 5th edn. Canada: Elsevier, 2017.

29 Germain AM, Blackmore AM, Gibson N, et al. Effects of adaptive Bungee Trampolining for children with cerebral palsy: a SingleSubject study. Pediatr Phys Ther 2019;31:165-74.

30 Breitenstein SM, Gross D, Garvey C, et al. Implementation fidelity in community-based interventions. Res Nurs Health 2010;33:164-73.

31 Brian A, Goodway JD, Logan JA, et al. SKIPing with teachers: an early years motor skill intervention. Phys Educ Sport Pedagogy 2017;22:270-82.

32 Sekhon M, Cartwright M, Francis JJ. Acceptability of healthcare interventions: an overview of reviews and development of a theoretical framework. BMC Health Serv Res 2017;17:13.

33 Braun V, Clarke V. Using thematic analysis in psychology. Qual Res Psychol 2006;3:77-101.

34 Blank R, Barnett AL, Cairney J, et al. International clinical practice recommendations on the definition, diagnosis, assessment, intervention, and psychosocial aspects of developmental coordination disorder. Dev Med Child Neurol 2019;61:242-85.

35 Eldridge SM, Lancaster GA, Campbell MJ, et al. Defining feasibility and pilot studies in preparation for randomised controlled trials: development of a conceptual framework. PLoS One 2016;11:e0150205.

36 Orsmond GI, Cohn ES. The distinctive features of a feasibility study: objectives and guiding questions. OTJR-Occup Part Heal 2015;35:169-77.

37 Henderson SE, Sugden DA, Barnett AL. Movement assessment battery for children-2 second edition (Movement ABC-2). London, UK: The Psychological Corporation, 2007.

38 Brown T, Lalor A. The Movement Assessment Battery for Children-Second Edition (MABC-2): a review and critique. Phys Occup Ther Pediatr 2009;29:86-103.

39 Dwyer GM, Hardy LL, Peat JK, et al. The validity and reliability of a home environment preschool-age physical activity questionnaire (Pre-PAQ). Int J Behav Nutr Phys Act 2011;8:86-98.

40 Carswell A, McColl MA, Baptiste S, et al. The Canadian occupational performance measure: a research and clinical literature review. Can $J$ Occup Ther 2004;71:210-22.

41 Goodman A, Goodman R. Strengths and difficulties questionnaire as a dimensional measure of child mental health. J Am Acad Child Adolesc Psychiatry 2009;48:400-3. 
